Itinéraires Itinéraires

Littérature, textes, cultures

2011-3 | 2011

Récits du corps au Maroc et au Japon

\title{
Yoshiyuki Junnosuke : voix et silence à travers le corps
}

Akira Hamada

\section{OpenEdition}

Journals

Édition électronique

URL : http://journals.openedition.org/itineraires/1537

DOI : $10.4000 /$ itineraires. 1537

ISSN : 2427-920X

Éditeur

Pléiade

\section{Édition imprimée}

Date de publication : 1 novembre 2011

Pagination : 117-126

ISBN : 978-2-296-55720-8

ISSN : 2100-1340

Référence électronique

Akira Hamada, "Yoshiyuki Junnosuke : voix et silence à travers le corps », Itinéraires [En ligne], 2011-3 | 2011, mis en ligne le 01 novembre 2011, consulté le 19 avril 2019. URL : http://

journals.openedition.org/itineraires/1537; DOI : 10.4000/itineraires.1537

\section{(ब) $(\Theta \Theta$}

Itinéraires est mis à disposition selon les termes de la licence Creative Commons Attribution - Pas d'Utilisation Commerciale - Pas de Modification 4.0 International. 


\title{
Yoshiyuki Junnosuke : voix et silence à travers le corps
}

\begin{abstract}
This article highlights the bodies described in the novels and short-stories of Yoshiyuki Junnosuke. The writer, allergic and sensitive, offers weak and damaged bodies of marginal people (prostitutes, tired man, etc.) with very delicate descriptions. The change of Japanese society influences the states of the bodies of his novels. The body of prostitutes shows their energetic power in the town, which is only possible in the Japan beaten after the World War II, free of traditional moral. It disappears shortly. As the Japan normalizes, the weak and damaged bodies are destined to be cooped up indoors.
\end{abstract}

Keywords : Yoshiyuki Junnosuke, prostitutes, town, Japan, illness

Mots clés : Yoshiyuki Junnosuke, prostituées, ville, Japon, maladie

Yoshiyuki Junnosuke (1924-1994) est connu pour ses romans centrés sur les rapports amoureux et la description minutieuse du corps des femmes à la différence des écrivains de la première génération de l'après-guerre qui sont plus politiques et engagés. Sa vie correspond approximativement à l'ère Showa (1926-1989) et la plupart de ses œuvres ont pour cadre cette époque caractérisée d'abord par la guerre du Pacifique et la défaite qui suivit, et ensuite par le développement économique. Notre article s'intéressera d'abord à l'écrivain qui est très sensible aux réactions de son corps à cause de ses maladies (asthme, dépression, allergie, etc.). Les représentations du corps seront analysées dans les récits des années 1950-1980, à travers de nombreuses citations.

\section{Yoshiyuki ou l'enfant malade}

Sa vie est une succession de maladies. L'allergie le tourmente toute sa vie et joue un rôle décisif sur la formation de sa sensibilité, très délicate. La typhoïde (16 ans) l'a profondément changé. Pendant sa convalescence, le brillant élève, redoublant, a trouvé dans la littérature une valeur essentielle pour lui. Il a même inventé une maladie, le syndrome asystolique dû au béribéri, pour quitter son 
lycée dominé par l'atmosphère militaire. L'asthme découvert trois jours après l'appel (20 ans) lui a permis de retrouver le monde civil. La dispense du service militaire n'est qu'une chance inespérée pour Yoshiyuki à la différence de Mishima qui aura du mal à la mettre en concordance avec ses idées patriotiques.

Après la guerre, comme la plupart des Japonais, sa vie n'était pas facile. Il a dû arrêter ses études à l'université de Tôkyô, et il a travaillé dans une maison d'édition comme journaliste d'une revue populaire. Le surmenage au travail est la cause de sa tuberculose pulmonaire (28 ans) selon Yoshiyuki. Il a dû arrêter son travail. Heureusement la tuberculose n'était plus une maladie incurable grâce à un nouveau traitement. Ses jours à l'hôpital ne ressemblaient plus à ceux, calmes et monotones, au sanatorium. De nombreux auteurs de la génération précédente ont évoqué le traitement de leur tuberculose, véritable maladie des écrivains. Après l'ablation d'une partie de son poumon gauche (30 ans), la vie quotidienne, partagée dans la même chambre d'hôpital que vingt-trois autres patients lui a permis de côtoyer de près des gens de classes différentes, et d'imaginer la vie de régiment qu'il n'a pas connue. C'est au cours de son hospitalisation qu'il a reçu un prix littéraire (prix Akutagawa) et qu'il a décidé de vivre de sa plume.

\section{Chanson enfantine (1961) ou l'enfant malade}

Voici un extrait de la Chanson enfantine (1961), récit d'un garçon hospitalisé. Le nom de la maladie n'est pas précisé, mais le mot « fièvre » se référerait à la typhoïde qui avait cloué le jeune Yoshiyuki au lit :

La fièvre ne descendit pas. Pour que la fièvre reste si élevée, il fallait de l'énergie. La chair et le sang du garçon furent utilisés comme énergie. La chair du tronc, celle des bras et celle des jambes disparurent subitement.

Pourtant, la fièvre élevée ne quitta pas son corps. La fièvre causa la perte de la chair du nez et celle couvrant le crâne et celle des gencives. La chair était tellement fine qu'un doigt pouvait toucher la ligne séparant deux parties du crâne. Les os des genoux saillaient comme s'ils étaient des boules malades formées sous les branches fines d'un pin. Les fesses perdirent de la chair et l'anus saillait comme un tube long. [...]

Le garçon se sentit glisser et tomber du monde des vivants.

«Allez, debout et fais des exercices de marche », ordonna le médecin. Le garçon entendit ces mots comme un ordre absurde. Il regarda le visage du médecin. Ce visage était vigoureux et gras. Il avait cru que ce visage était celui qui donnait l'ordre. Il avait interprété ainsi la vigueur et la graisse luisante sur le visage.

Pourtant quand il regarda le visage de nouveau, il se dit : «C'est juste le visage des vivants. "

Son ami et le médecin étaient debout sur le même plan. Lui seul commençait à tomber de ce plan ${ }^{1}$.

1. Yoshiyuki Junnosuke, Dôyô [Chanson enfantine] [1961], Euvres complètes, t. II, Tôkyô, Shinchôsha, 1997, p. 329-331. Nous traduisons. 
La description du corps ravagé par la maladie est certes horrible, mais en même temps objective et anatomique et ne mentionne pas la douleur. Non seulement la maladie et la fièvre transforment le corps, mais elles emmènent l'enfant jusqu'à la frontière du monde des vivants. L'enfant tirera des enseignements utiles de cette expérience pénible : enfant, il sait déjà que la vie est très précieuse, mais fragile.

\section{La Fleur bleue (1959) ou l'écrivain malade}

Le personnage du récit suivant est un écrivain malade, double de Yoshiyuki. En effet, cet écrivain souffrit d'allergie toute sa vie.

Pendant ces six mois, il ne sortit jamais de la maison. Il n'avait pas la force de sortir.

Chaque matin, en se réveillant, il sentait comme s'il y avait un peu de liquide au fond d'un petit récipient dans son corps. Le liquide était son énergie physique pour la journée. Il consacra tout ce liquide à l'écriture. Il mena ainsi sa vie difficilement. Assis devant le bureau, il se rendit compte que ses cellules perdaient de l'eau et se desséchaient.

En effet, au fur et à mesure que les heures passaient, sa peau se flétrit et des rides apparurent.

Ce matin-là, il lui sembla que la quantité de liquide était plus importante que d'habitude. Comme il avait une réunion à ne pas manquer, il sortit de chez lui pour la première fois depuis six mois.

Dès le moment où il entra dans la salle de réunion, il resta paralysé. L'odeur du santal blanc y flottait imperceptiblement. Cette odeur était dangereuse pour lui. Déjà, l'odeur pénétrait en spirale dans chaque cellule des tubes entre les fosses nasales et les poumons. [...]

Les spirales innombrables vinrent mordre ses cellules. Le bouchon de liège se déboucha, et les mucosités suintèrent abondamment dans les fosses nasales et les tubes bronchiques.

S'échappant pendant la réunion, il retourna chez lui en vomissant des liquides transparents et muqueux. Ses cellules, desséchées ou débordantes, ne se stabilisèrent pas à l'état adéquat.

Une fois rentré chez lui, il se laissa tomber sur son lit. Les heures passèrent, les sécrétions s'apaisèrent mais la fatigue resta intense ${ }^{2}$.

L'allergie n'est pas une maladie contagieuse causée par un virus ou un microbe, mais une réaction anormale, excessive du système de l'organisme avec une substance étrangère. Le personnage de ce récit ne peut jeter le blâme sur la substance, ici le santal blanc, qui n'est pas nocif. Il doit être vigilant vis-à-vis du monde extérieur qui peut déclencher une réaction inadaptée et être conscient de son corps.

Ce qui attire notre attention, c'est l'abondance des expressions physiologiques comme « cellules » ou « sécrétions ». Ces mots ne sont pas utilisés

2. Yoshiyuki Junnosuke, Aoi hana [La Fleur bleue] [1959], Euvres complètes, op. cit., t. II, p. 107-108. Nous traduisons. 
d'une façon scientifique à proprement parler. Sensible, le narrateur a recours à ces expressions pour expliquer sa réaction physique alors que la plupart des gens n'ont pas besoin de préciser ce qui se passe au fond de leur corps.

L'hypersensibilité n'entraîne pas seulement une réaction pénible et négative, mais contribue aussi à la création de l'espace intérieur de son corps.

\section{Le Japon moderne et le corps}

Nous reprendrons ici quelques remarques historiques sur les corps japonais, notamment celles du critique Yoro Takeshi ${ }^{3}$.

Selon lui, les corps japonais ont suivi des règles qui contrôlaient les mouvements physiques. Par exemple, une fois la paix établie sous le shogunat, les samourai (guerriers) ne se battent plus sur les champs de bataille, ils deviennent fonctionnaires dans un système assez hiérarchisé. Même s'ils continuent de prendre le sabre, ce n'est plus pour tuer des adversaires mais s'entraîner physiquement et moralement dans l'exercice. L'entraînement prendra l'appellation kenjutsu (art du sabre), avant de devenir kendo (voie du sabre). Le corps et les mouvements sont souvent codifiés et conditionnés par des règles strictes.

L'effondrement du régime du shogunat en 1868 eut pour conséquence l'abolition de systèmes qui avaient réglementé les comportements des Japonais. Les modèles occidentaux qui furent introduits alors plongèrent le pays dans le chaos esthétique alors que le résidu des formules conventionnelles continuait de conditionner les corps japonais.

Les écrivains de l'ère Meiji s'affrontent au sujet du modernisme. Natsume Sôseki insiste sur la nécessité de l'établissement de l'individu, une réalité qui ne s'enracine pas au Japon. Le titre de son œuvre principale est justement Kokoro (cœur). Le titre retenu en français a été Le Pauvre cour des hommes. Mori Ôgai choisit de rechercher son modèle dans l'histoire.

Depuis les années 1930, la monté du militarisme et celle du nationalisme présentent un nouveau modèle du corps. Les hommes doivent s'offrir comme soldats pour la patrie. Les corps des soldats sont idéalisés avec leurs uniformes militaires.

Après la guerre, c'est de nouveau le chaos. Les écrivains de la première génération de l'après-guerre traitent le corps dans les champs de bataille (Ooka Shohei) et dans le monde d'une armée hiérarchisée et inhumaine (Noma Hiroshi).

Comme nous l'avons dit plus haut, Yoshiyuki n'a pas participé à la guerre comme soldat, ce qui lui a épargné de suivre leur modèle. Sa maison a été incendiée par le bombardement. Yoshiyuki, malade et pauvre mais libre, commence alors à écrire des récits dans lesquels les personnages font l'expérience du chaos de l'après-guerre.

3. Yoro Takeshi, Shintai no bungakushi [L'Histoire littéraire du corps] [1997], Tôkyô, Shinchôsha, 2001. Anatomiste de formation, Yoro publie aussi des essais critiques. 


\section{Les corps dans les récits de Yoshiyuki}

\section{Le choix de la transcription du mot corps selon Yoshiyuki}

Il existe plusieurs idéogrammes pour désigner le corps en japonais. Par exemple, 体 ou 身体 sont les plus courants. Yoshiyuki n'utilise pas ces idéogrammes au moins pour désigner les corps de ses personnages. Pour lui, ces idégrammes font penser à des corps sains et forts, par exemple ceux de gymnastes. Ces corps sont beaux et parfaits, mais satisfaits d'eux-mêmes et sans complexes. Peu sensibles, ils résistent aux forces extérieures.

L'idéogramme que Yoshiyuki préfère est 軀 qu'on trouve rarement chez d'autres écrivains. Il explique la raison de son choix. Son explication n'est pas étymologique mais vient de son impression personnelle. Cet idéogramme signifie pour lui un beau corps abîmé et affaibli, par exemple celui de la prostituée ${ }^{4}$.

\section{La Ville aux couleurs fondamentales (1956)}

Après la guerre, le gouvernement autorise la prostitution dans certains quartiers bien délimités. Ces quartiers ont pris l'appellation d'akasen (ligne rouge). Ils furent édifiés sur des terrains qui venaient d'être dévastés par la guerre et se situaient aux antipodes de la vieille image traditionnelle du yûkaku (quartier de maisons closes) de l'avant-guerre, cher en particulier à Nagai Kafu.

Voici la description d'un de ces quartiers et des prostituées que le narrateur présente au début du récit. L'année de l'histoire est précisée dans le texte original, la vingt-cinquième année de l'ère Shôwa (1950). Il est à noter que le procédé de datation n'est pas habituel dans les récits de Yoshiyuki. Ce procédé témoigne d'une intention particulière : décrire l'époque de l'immédiat après-guerre.

Des pièces de tissu rose pendent à l'entrée des maisons à l'occidentale, devant lesquelles sont posées des filles aux lèvres et aux ongles écarlates. Dans des tenues étudiées, elles s'évertuent à attirer les regards et décochent aux passants de luisantes œillades savamment travaillées. La caresse mielleuse de leur voix au timbre nasal s'insinue jusqu'à eux; certaines les abordent de front pour les enlacer et, pressant contre eux leur ventre rebondi, leur glissent à l'oreille quelque obscénité.

Les corps qui font dans le charme discret sont plutôt rares et risquent de ne pas trouver preneur ${ }^{5}$.

4. Miyagi Mariko, Junnoshuke san no koto [À propos de mon cher Junnosuke], Tôkyô, Bungeishunjû, 2003, p. 54. Yoshiyuki conseille à sa compagne Miyagi d'éviter l'utilisation d'un idéogramme qui ne lui va pas.

5. Yoshiyuki Junnosuke, La Ville aux couleurs fondamentales [1956], dans L'Averse et La Ville aux couleurs fondamentales, trad. Pierre Deveaux, Arles, Picquier, 1995, p. 36. 
Voilà des femmes qui veulent être une marchandise attirante dans un cadre criard. Dans cette description du début du récit, les corps maquillés et colorés des prostituées sont fragmentaires et confondus dans le quartier sans que leur corps soit intégralement décrit. Le personnage principal de ce récit est une prostituée qui s'appelle Akemi. Elle a un corps discret mais « qui était parvenu à découvrir le sentiment de délivrance ${ }^{6}$ ». Dans ce quartier, la rencontre avec un jeune homme (Motoki) la mettra dans un état d'esprit instable :

Sur son visage de profil [Ruriko], la peau retenait le regard par sa blancheur et sa souplesse manifeste; une pâleur un peu maladive, pensa-t-il [Motoki]. Subitement, elle lui rappela Haruko, la petite prostituée qui revenait si souvent dans les bavardages de Gorô Mochizuki. Mais une fille comme Haruko, c'était fatal, ne pouvait qu'être marquée physiquement par les fatigues de sa profession, de l'argent, de son milieu. Sa compagne, en revanche, dans son enveloppe de peau précieusement préservée de toute souillure, gardait intacte sa beauté : son entourage y veillait, en vue de ce qui serait la seule et unique transaction de sa vie, à savoir son mariage ${ }^{7}$.

Ce jeune homme fait connaissance d'une jeune fille (Ruriko) dans le cadre du mariage arrangé avant de connaître Akemi, l'héroïne. Il trouve une ressemblance entre Ruriko et une prostituée (Haruko) qui fait partie du groupe des prostituées évoquées précédemment. Elles n'ont pas de points communs physiquement, mais toutes les deux sont considérées comme une marchandise achetée et vendue dans la société. Une fois qu'il connait bien Akemi et son caractère, il pense même que c'est Ruriko, venant d'une bonne famille, qui convient mieux à ce quartier de plaisir qu'Akemi, qui fait dans le charme discret et qui a l'esprit fin. Pourtant Akemi verra son beau corps s'abîmer et s'affaiblir. Le récit constitue donc une critique indirecte de la morale hypocrite du Japon à travers les corps des prostituées. En 1958, deux ans après la publication du récit, l'akasen est aboli et les couleurs fondamentales de la ville commencent à s'estomper.

\section{La barque du lit (1958) ou les corps au bord de l'épuisement}

Le narrateur-personnage commence par dire qu'il était « au bord de l'épuisement ${ }^{8} \gg$ avant de dire qu'il était professeur d'anglais dans une école pour jeunes filles. Il gagne difficilement sa vie et l'expression de « au bord de l'épuisement » apparaît trois fois en deux pages. Un soir, il fait la connaissance de Misako, un homme prostitué qui avait souhaité devenir une femme. Il écoute le récit monotone de Misako et la douceur de ses attentions s'infiltre en lui.
6. Ibid., p. 38 .
7. Ibid., p. 56.
8. Yoshiyuki Junnosuke, «La Barque du lit» [1958], dans Anthologie de nouvelles japonaises contemporaines, t. II, Le Désir, trad. Pascale Simon, Monaco, Éditions du Rocher, p. 53. 


\section{Il s'interroge sur sa douceur :}

J'avais une vague idée sur la nature de cette douceur. Je sentis qu'elle se manifestait parce qu'elle tentait, au bord de l'épuisement, d'aiguillonner ses dernières forces vitales afin de devenir une femme. Cette pensée se précisa quand elle me montra une photo de nu.

C'était une photo d'elle en plan moyen, nue. Elle avait les bras croisés sur la poitrine et souriait en penchant légèrement la tête.

- Alors, j'ai l'air d'une femme et de rien d'autre, non?

- À combien d'années remonte cette photo?

Mais j'avalai cette question. Sur la photo, le contour de son visage et les lignes de son corps étaient ronds et doux, et elle ressemblait à s'y méprendre à une femme. Or ces lignes rondes et douces étaient en train de disparaître du corps de celle qui était en face de moi ${ }^{9}$.

L'homosexualité ne le tentant pas, il ne peut pas aimer physiquement Misako. Il chérit sa douceur, elle qui, comme lui, est au bord de l'épuisement. La tendresse de Misako vient de la difficulté insurmontable dont il prévoit le dénouement inévitable.

\section{Oiseau, bête, insecte ou poisson (1959)}

Le début du récit exprime l'état d'esprit du narrateur-personnage d'une façon très impressionnante. À ses yeux, tous les objets de la ville perdent de leur propre couleur, en contraste avec la ville aux couleurs fondamentales qui est très animée par les désirs charnels :

À cette époque, le paysage de la ville me paraissait teinté de la couleur du plâtre. Le tramway, qui roulait lentement avec une perche saillante était un insecte de la couleur du plâtre. Les voitures, qui se traînaient par terre étaient aussi des insectes couverts de carapaces dures de la couleur du plâtre.

Non seulement ces machines, mais aussi les gens que je croisais dans les rues, et ceux que je rencontrais au hasard dans un coin de la ville, tous commençaient à se déformer, à perdre leurs couleurs et devenaient des objets étrangers à la couleur de plâtre.

Tantôt ils allongeaient leur cou, tantôt ils faisaient saillir leurs lèvres, gonfler les bras, et ils prenaient tout d'un coup une forme bizarre et étrangère. Chacun d'eux ressemblait à quelque oiseau, bête, insecte ou poisson mais restait toujours indéfinissable. C'était quelque chose qui n'avait aucun lien avec moi-même dans ma mémoire.

Chaque matin, je traversais ces rues avant d'arriver au bureau ${ }^{10}$.

Quel paysage intérieur! La description est poétique mais très sèche. L'auteur exprime ainsi l'aliénation du personnage principal au milieu des gens ordinaires qui mènent leur vie quotidienne. Un psychiatre

9. Ibid., p. 61.

10. Yoshiyuki Junnosuke, Tori kemono uo mushi [Oiseau, bête, insecte ou poisson] [1959], Euvres Complètes, op. cit., t. II, p. 60. Nous traduisons. 
diagnostiquerait une maladie mentale, par exemple la dépression ${ }^{11}$. De retour du bureau, un jour, il rencontre de façon imprévisible une jeune fille qui lui semble avoir la forme et le visage d'un être humain. Cette rencontre lui permet de retrouver le sens de la vie. Tout en lui montrant de la sympathie, elle refuse de le recevoir chez elle. En effet, elle a subi une opération à cause de la tuberculose et a peur de le dégoûter en montrant son corps déformé. Il l'a pourtant aimée comme elle était, avec ce corps dont elle avait honte :

Je veux guérir ta blessure.

Et j'ai secoué son corps plus violemment. Elle s'est agitée dans mes bras.

À ce moment, soudain a retenti un bruit sourd. Des notes bizarres comme celles produites quand on ouvre longuement et avec force le soufflet troué d'un accordéon.

Elle a caché son visage dans mes bras et a murmuré.

« C'est mon poumon qui fait du bruit. Comme on m'a enlevé un os, quand je bouge brusquement, ça fait ce bruit-là. »

Attendri, j'ai donné affectueusement encore une fois un baiser sur sa grosse cicatrice. J'ai appliqué un baiser fort pour qu'il puisse toucher son autre blessure cachée au fond de sa cicatrice.

Pourtant, ce jour-là non plus, son corps ne s'est pas ébranlé sous le mien. J'ai rêvé que son corps se tortillait sous le mien, que mon cœur enveloppait son corps tendrement et que le bruit de son poumon retentissait. J'ai rêvé que le bruit sourd et un peu bizarre résonnait solennellement comme une trompette de victoire. Pourtant cela ne s'est pas produit. Notre voyage venait de débuter ${ }^{12}$.

Très rare est le lyrisme si touchant de la fin de ce récit parmi les œuvres de Yoshiyuki dont le style est assez retenu. Le regain d'humanité du personnage est d'autant plus réjouissant qu'il se réalise avec un corps abîmé et déformé. Le bruit de son poumon ne serait pas agréable à l'oreille mais il n'est autre chose que le cri de la vie.

\section{La Chambre noire (1970) ou les femmes ambiguës}

Nakata, narrateur-personnage de ce roman, est un écrivain d'âge mûr. Il vit tout seul et fréquente peu les gens. Avec de nombreuses digressions, l'intrigue du roman n'est pas facile à résumer mais on peut dire que l'évolution des relations compliquées avec ses maîtresses occupe une place centrale. Le héros ne cherche pas de relations amoureuses, préférant des rapports purement sexuels.

Quand il fait connaissance avec Maki, il ne sait pas qu'elle est lesbienne. Devant le refus de Maki, il essaie de la conquérir :

11. Dans le résumé chronologique de sa vie, Yoshiyuki mentionne une dépression grave en 1967, à l'âge de 43 ans. Yoshiyuki connaîtra plusieurs moments de dépression au cours de sa vie.

12. Ibid., p. 88-89. 
En entendant ces mots, je pris soudain conscience de sa nudité. Elle n'avait plus sur elle que le haut de ses vêtements. Je me sentis excité par un désir trouble. Je l'attaquai une seconde fois, par surprise, et comme elle opposait encore une certaine résistance, je la fis basculer après l'avoir saisie à bras-le-corps.

Je plaquai de nouveau mon visage sur son ventre. Je me retrouvai alors à peu près dans la même position que précédemment et fus frappé d'un seul coup par le comique de la situation.

Maki se débattait et cherchait à s'enfuir; de mon côté, j'essayais de la retenir mais je ne trouvais pas dans mes bras la force nécessaire. Bientôt, elle me souffla :

- Bon, allez, on arrête?

À ces mots, toute ardeur me quitta. Dès le début, elle n'avait eu à mes yeux que peu d'attrait physique. Pensant, comme elle, qu'il valait mieux s'en tenir là, je desserrai mon étreinte.

- C'est étrange, mais je n'ai pas eu la nausée, marmonna Maki, comme pour elle-même ${ }^{13}$.

Pourquoi a-t-elle parlé de nausée? C'est après leur première relation réussie qu'il se rend compte de la signification de la question. Il comprend que Maki est lesbienne. Nakata lui pose des questions pour la comprendre et comprendre l'homosexualité féminine. Ils échangent leurs idées mais le mystère reste jusqu'à la fin. Maki le quitte en disant qu'elle ira accoucher de leur bébé à New-York et y élever leur enfant toute seule. Ses autres maîtresses entretiennent des relations ambiguës avec Nakata. L'une d'entre elles, Takako, professeur d'art d'arrangement floral, a attendu quatre ans avant de le quitter pour se marier. Yumiko, sa femme de ménage, l'a quitté aussi pour se marier. Finalement c'est avec Natsué qu'il entretient une relation durable. Absorbée purement par le plaisir sexuel, Natsué ne s'intéresse pas au mariage, ce qui met Nakata à l'aise. Lectrice d'Histoire d'O, elle introduit une relation maître-esclave, qui fascine et fatigue Nakata. Après un mois passé avec Natsué, il ne peut pas s'empêcher de la voir.

Quand je pénétrai dans l'immeuble, je perçus, flottant à peine dans l'air, l'odeur de Natsue. Son corps, à présent, n'avait plus d'odeur. Pourtant, il y avait encore dans l'air cette odeur presque imperceptible qui m'excitait et m'attristait à la fois. Je montai l'escalier et m'avançai le long du couloir en béton. L'odeur se faisait de plus en plus intense. Sans doute étais-je le seul à la sentir. Bientôt, elle devint violente, au point de me suffoquer les narines.

À cet instant, j'arrivai devant chez elle. Je saisis le loquet de la porte. Au-delà, c'était la chambre noire ${ }^{14}$.

Le roman contient des anecdotes assez hétérogènes dont certaines remontent dans son passé : le doute quant à l'adultère de sa femme morte avec son ancien ami, et l'histoire d'une fille mentalement attardée et cachée dans les combles, qui était la sœur d'un génie scientifique. Il n'empêche que

13. Yoshiyuki Junnosuke, La Chambre noire [1970], trad. Yuko Brunet et Sylvie H. Brunet, Arles, Picquier, 1990, p. 29.

14. Ibid., p. 219 
les corps des maîtresses de Nakata se débarrassent des traces de l'aprèsguerre, la pauvreté et le sentiment d'usure n'existant pas. Les corps des femmes que le personnage fréquente ne s'exposent plus aux regards du monde mais sont enfermés dans leurs relations avec Nakata.

\section{En guise de conclusion}

Beaucoup de formules conventionnelles conditionnent les esprits et les comportements à l'ère d'Edo. Comme la Restauration de Meiji a aboli l'ancien système, la défaite de la guerre du Pacifique a bouleversé les Japonais et entraîné un état chaotique dans leurs comportements.

Ceux qui ne peuvent pas supporter la dégradation de la morale ont essayé de restaurer les formules traditionnelles et fait renaître l'esthétique classique. Mishima représente le cas extrême de ces traditionalistes.

L'uniforme militaire symbolise le sacrifice réclamé par l'esprit communautaire. Si la cause du patriotisme privilégie l'uniforme militaire et les corps virils des soldats, le corps de Yoshiyuki se situe aux antipodes de ce qu'exige le patriotisme. Les maladies auraient permis à Yoshiyuki d'acquérir une sensibilité délicate. L'analyse psychologique se réalise par les métaphores utilisant un lexique physiologique, notamment les cellules et les liquides. Son choix de l'idéogramme du corps 軀 est assez significatif pour comprendre le sens, dans ses récits, d'un corps abîmé et affaibli.

Les personnages de Yoshiyuki sont souvent marginaux dans la société japonaise et leurs corps intimes et personnels, ne sont pas représentatifs de ceux des Japonais. La lecture de ses récits révèle cependant des changements historiques, et montre en filigrane le reflet du Japon des années 1950-1980.

Dans le Japon chaotique qui caractérise la fin de la guerre, les corps des prostituées ont brillé avec leur vitalité dans « la ville aux couleurs fondamentales », libérés de la morale sociale. Une fois que l'ordre est revenu, les rues sont remplies des gens ordinaires, qui semblent « oiseau, bête, insecte ou poisson » aux yeux du personnage de Yoshiyuki. Fatigués, les personnages chechent du repos dans la «barque du lit» et ceux qui ne peuvent pas accepter des relations amoureuses normales se renferment dans la « chambre noire ». C'est ainsi que le corps subit une intériorisation causée par la normalisation de la société japonaise.

Ce qui est essentiel dans les récits de Yoshiyuki, c'est l'état d'esprit dans la relation entre les personnages. Ces personnages, sensibles et fragiles ne se veulent pas éloquents. Or, c'est à travers leurs corps qu'ils nous permettent d'entendre des voix originales. 